\title{
A FUGAZ VISITA DE JORGE LUIS BORGES A MEDELLÍN EM $1965^{1}$
}

\section{LA VISITA FUGAZ DE JORGE LUIS BORGES A MEDELLÍN EN 1965}

Rodrigo de Jesus Garcia Estrada ${ }^{2}$

UdeA: https://orcid.org/0000-0002-7554-7006

Andrés López Bermúdez

UdeA: https://orcid.org/0000-0002-6634-2252

DOI: $10.21680 / 1982-1662.2021 v 4 n 30 I D 22414$

\section{Resumo:}

Jorge Luis Borges, um dos escritores latino-americanos mais universais, esteve em Medellín em 1965, um ano após ter sido forte candidato ao Prêmio Nobel de Literatura, e fez parte do intercâmbio cultural entre Colômbia e Argentina, cujos governos retornaram, com medo e incertezas, ao regime democrático, após vários anos de ditadura. Foi um périplo do escritor argentino como homem público, que em poucos dias visitou quatro cidades colombianas. Sua passagem por Medellín foi rápida, mas conseguiu convidar os medellinenses a fazer parte de seu círculo de leitores e admiradores. Este artigo reconstitui as atividades realizadas por Borges na capital do departamento de Antioquia, no marco de um cenário definido por circunstâncias políticas, culturais e diplomáticas.

\footnotetext{
${ }^{1}$ Artículo resultado de la investigación "Notas histórico-literarias sobre la primera visita de Jorge Luis Borges a Medellín (1965)", realizado con el aval del Consejo de la Facultad de Ciencias Sociales y Humanas y el apoyo financiero de la Universidad de Antioquia. Inscrito en el Sistema Universitario de Investigación, con el código 2018-19530, con una duración de seis (6) meses.

${ }^{2}$ E-mail: rodrigo.garcia@udea.edu.co
} 
Palavras-chave: Jorge Luis Borges. Viagens. Diplomacia cultural. História intelectual. Literatura argentina. Imigração argentina.

\section{Resumen:}

Jorge Luis Borges, uno de los escritores latinoamericanos más universales, estuvo en Medellín en 1965, un año después de ser firme candidato al premio Nobel de Literatura, e hizo parte del intercambio cultural entre Colombia y Argentina, cuyos gobiernos retornaron con temor e incertidumbre al régimen democrático, después de varios años de dictadura. Se trataba de un periplo como hombre público del escritor argentino, quien recorrió en pocos días cuatro ciudades colombianas. Su paso por Medellín fue raudo, pero logró invitar a los medellinenses a hacer parte de su círculo de lectores y admiradores. Este artículo reconstruye las actividades realizadas por Borges en la capital antioqueña, en el marco de un escenario definido por circunstancias políticas, culturales y diplomáticas.

Palabras clave: Jorge Luis Borges. Viajes. Diplomacia cultural. Historia intelectual. Literatura argentina. Inmigración argentina.

\section{Abstract:}

Jorge Luis Borges, one of the most universal Latin American writers, was in Medellín in 1965, a year after being a firm candidate for the Nobel Prize for Literature, and was part of the cultural exchange between Colombia and Argentina, whose governments returned with fear and uncertainty to the democratic regime, after several years of dictatorship. It was a journey as a public man of the Argentine writer, who traveled in a few days to four Colombian cities. His time in Medellín was swift, but he managed to invite the people of Medellín to become part of his circle of readers and admirers. This article reconstructs the activities carried out by Borges in the Antioquia capital, within the framework defined by political, cultural and diplomatic circumstances. 
Key words: Jorge Luis Borges, travel, cultural diplomacy, intellectual history, Argentine literature, Argentine immigration.

\section{Introducción}

Jorge Luis Borges viajó a Colombia en julio de 1965, como invitado de una Misión cultural cuya presencia fue solicitada por el embajador argentino en Bogotá, Sr. Arturo Mathov (RAMÍREZ, 1965), realizada en el marco de las relaciones diplomáticas, económicas y sociales entre ambas naciones y siguiendo una directriz expresa del Ministerio de Relaciones Exteriores de su país (ROMERO, 1965a; PARDO, 1965). Precisamente, en 1964 se firmó en Bogotá un Convenio de Intercambio Cultural, y con la visita de la delegación argentina dicho acuerdo empezó a materializarse. Para llevar a cabo su propósito, el embajador argentino se apoyó en las nacientes colonias de ciudadanos argentinos asentadas en las principales capitales colombianas, y el programa en su conjunto se denominó “Mes de la amistad Colombo-Argentina” (SIN AUTOR, 1965f).

Es oportuno mencionar que el mes y la fecha elegidas para dicha visita obedecieron a la intención de fortalecer los lazos de hermandad entre ambas naciones, rememorando el apoyo de los voluntarios argentinos a las tropas bolivarianas que le dieron la libertad a Colombia, y la celebración de la fiesta nacional de Argentina del 9 de julio. Por su parte, Borges sentía mucho respeto por la guerra fundacional de Colombia, dado que un abuelo suyo fue comandante de caballería en la batalla de Junín (GIRALDO, 1965).

En dicho contexto se entiende que la delegación cultural argentina que visitó a Colombia, se encontró acompañada por una representación de las Fuerzas Armadas del país del sur, integrada por miembros del Regimiento de Granaderos a Caballo "General José de San Martin" (equivalente a la Guardia Presidencial de Argentina), cuerpo comandado por el teniente coronel Ulises Muschietti, jefe de la delegación castrense, a quien secundaron el capitán Oscar Augusto Blanco y el teniente Agustín Vásquez Arrubarena, cinco suboficiales y ocho soldados de la citada unidad. Complementariamente, en representación del Regimiento Número 1 de Infantería 
“Patricios”, viajaron el capitán Alejandro Lemme, un suboficial y cuatro soldados.

\section{Notas para un estado del arte}

La presencia en Medellín, en 1965, de uno de los grandes escritores de la literatura universal, el argentino Jorge Luis Borges (Buenos Aires, Argentina, 1899 Ginebra, Suiza, 1986), no ha merecido el suficiente interés por parte de los historiadores, pero sí de los admiradores y practicantes del culto borgiano -como puede calificarse a quienes no sienten recato a la hora de elevar a este escritor a los altares y a la categoría de dios o profeta-. Hasta la fecha, este acontecimiento ha inspirado la pluma de escritores, poetas y periodistas colombianos, aunque con mayor énfasis de quienes fueron testigos de su estadía en el país cafetero a finales de 1978.

En 1965, Borges apenas era conocido en Colombia y sólo lo leían las minorías aburguesadas, según recordó en 2011 el poeta Elkin Restrepo, testigo de una conferencia que el escritor dictara en el Hotel Nutibara. Mientras que, durante su segundo paso por Medellín, hacia 1978, "su sólo nombre despertaba el delirio colectivo", dijo el bardo antioqueño, quien, describió la impresión que le causaron los gestos, silencios y momentos dubitativos del argentino durante las dos horas del conversatorio del 78; así como su insistencia en que no le llamaran "Maestro", sino, simplemente Borges, lo que permite observar su molestia con quienes insisten en deshumanizarlo. Y, al tener ocasión de verlo luego del evento, a solas en un rincón de la Biblioteca Pública Piloto, donde se realizó el encuentro ante muchos espectadores, lo representó con las siguientes palabras: “Pequeño, frágil, mortal, anhelante de estar consigo mismo, cumplido una vez más el papel de minotauro ciego e intimidante" (RESTREPO, 2011).

Otro testimonio de la presencia del argentino en Medellín es el libro Borges. Memoria de un gesto (OSORIO y BUENO, 1979), quienes presentan su relato personal sobre las circunstancias que les permitió conocerlo en Cartagena durante su tercer viaje a Colombia. Es claro que se trató de una extensa entrevista, que le resultó incómoda al escritor y alucinante a sus interlocutores, cuyo producto es un texto lleno de alabanzas desmedidas e imaginación desbordante. Pero gracias a estos jóvenes 
escritores fue posible la segunda visita del escritor a la capital antioqueña en noviembre de 1978, en la que, como un gesto de reconocimiento a su obra universal, el alcalde Jorge Valencia Jaramillo le entregó las llaves de la ciudad (OSORIO y BUENO, 2003). Además, el texto de Osorio y Bueno, reproduce una serie de documentos de primera mano, como la alocución del director de la Biblioteca Pública Piloto el día de la conferencia; varias columnas de prensa publicadas en medios de comunicación de Medellín como El Colombiano y El Mundo, y de otras ciudades colombianas, caso de Cartagena, Cali y Bogotá, en las que se destacó la presencia del ilustre argentino; y el discurso del escritor al momento de recibir las llaves de la ciudad (OSORIO y BUENO, 1979).

En años recientes son cuatro las reseñas periodísticas que se han publicado sobre la segunda visita del argentino a Medellín. La primera, de Dora Luz Echeverría, “La copa de Borges", publicada en Universo Centro en 2013, un texto literario que intenta recrear el paso de Borges por Colombia en 1978, en particular sus días en Bogotá y Cartagena, recreando anécdotas recogidas entre las personas que tuvieron oportunidad de cruzar palabras con el visitante. Echeverría, estuvo en la "comida" que le ofreció la Universidad de Antioquia, luego de su presentación en el Paraninfo, y le tocó sentarse a la izquierda de Borges, escuchar sus evocaciones de la ciudad amurallada y presenciar la larga charla del argentino con el escritor paisa Manuel Mejía Vallejo (ECHEVERRÍA, 2013).

Por último, entre el 13 y el 14 de junio de 2016, con motivo de la conmemoración de los 30 años de la muerte de Borges, aparecieron en la prensa tres artículos que evocan los pasos de esa “criatura fantástica” como le llamó la periodista Mónica Quintero en su crónica de El Colombiano, mientras que Héctor Abad Faciolince y Juan Camilo Rincón hicieron lo propio. El periodista Rincón en su artículo “El sueño que Borges dejó en Colombia”, publicado en El Espectador, narra cómo se aproximó Borges a algunos intelectuales colombianos que lo motivaron a venir al país en diciembre de 1963, gracias a Jorge Gaitán Durán y a su revista Mito que le dedicó dos números a su obra. También se refiere a su regreso a la capital colombiana el 7 de julio de 1965, y a un viaje proyectado para diciembre de 1977, cuando la alcaldía de Bogotá le otorgó al porteño la Orden Civil al Mérito “Ciudad de Bogotá”, a pesar de lo cual sólo pudo viajar a Colombia seis meses más tarde, en noviembre de 1978 . De 
soslayo, Rincón referencia la segunda visita a Medellín, destacando el libro antes citado de Osorio y Bueno (RINCÓN, 2016).

Por su parte, Abad Faciolince publicó en su blog un ameno texto intitulado “Borges: 25 años de inmortalidad”, en el que se declara admirador casi fanático de Borges: "Haberlo conocido, quiero decir, haberlo visto simplemente, le da a los propios ojos y a la propia memoria un cierto halo sagrado [...] Pues bien, todos los que vimos a Borges alguna vez, y así haya sido en una sola ocasión, guardamos la memoria de ese instante. Lo atesoramos, lo adornamos. Nos ufanamos” (ABAD, 2016). Aparte de exaltar la genialidad del argentino, Abad sólo refiere su fugaz encuentro con él: "En Medellín, donde vivo y donde todavía se venden como reliquias trocitos chamuscados de la guitarra de Gardel, Borges estuvo dos veces. La segunda vez yo estuve ahí (en la primera, pocos sabían que Borges era Borges, y los 20 presentes hoy se sienten ungidos por la gloria) y guardo como una de mis pocas medallas vitales el haberlo visto" (ABAD, 2016).

El más reciente texto sobre el tema de interés es de la periodista Mónica Quintero que, a partir de sus entrevistas a Darío Ruiz, Elkin Restrepo y Olga Elena Mattei, expone algunas anécdotas, recuerdos y palabras dichas por el argentino durante su estadía en Medellín en 1965. Esta crónica retrata a partir de testimonios el deslucido lugar del Hotel Nutibara donde unos pocos pudieron escucharlo y hacerle preguntas, para oír sus respuestas, que dejaban atónitos a los asistentes. En esa ocasión hubo además un conversatorio con Borges en el Paraninfo de la Universidad de Antioquia. Fue Elkin Restrepo quien le inspiró el título a la periodista, cuando dijo: "Yo acudí como el que va a ver a una criatura fantástica" (RESTREPO, 2011).

Como puede verse, los únicos interesados en estudiar las visitas de Borges a Colombia han sido escritores y periodistas, en su mayoría admiradores de la obra narrativa del porteño y varios de ellos estuvieron presentes en las charlas impartidas por él en Medellín. Se sabe más de la segunda visita, por el libro publicado por Osorio y Bueno, que recoge los recuerdos personales de éstos y los reportes de la prensa antioqueña. Intentar abordar de nuevo el tema desde el punto de vista histórico puede aportar una mirada diferente, contextual y a partir de fuentes documentales, así como indagar fuentes literarias, memorias, autobiografías y crónicas de ciudad. 


\section{El viaje y su contexto}

El rápido paso de Borges por Colombia en 1965 hizo parte de las labores político-culturales de la vida pública de los intelectuales, quienes, por convicción, o por mediación de sus mecenas y amigos, pueden ser instrumentalizados por los gobiernos de sus países o por las instituciones que les brindan apoyo. Por supuesto, no fue un viaje de turismo y tampoco se trataba de un peregrinaje para encontrarse con algunos de los lugares favoritos o tumbas de sus autores predilectos. $\mathrm{Y}$, por tratarse de un viaje enmarcado en la diplomacia cultural del gobierno argentino, es necesario ubicar el contexto histórico-político del país austral y de Colombia, así como otros factores que contribuyeron a su realización.

De un lado, se debe establecer que Colombia, luego de trasegar por la dictadura de Gustavo Rojas Pinilla, la cual fracasó en su intento por "pacificar" el país, se encontraba bajo el pacto del Frente Nacional, por medio del cual los partidos liberal y conservador se repartieron el poder, excluyendo a terceras fuerzas políticas, legitimando, sin pretenderlo, la formación de guerrillas de izquierda (GUZMÁN, FALS y UMAÑA, 1962 y 1964). El presidente de la República era el abogado Guillermo León Valencia, quien puso su empeño en acabar -sin lograrlo- las guerrillas y sus “repúblicas independientes” (ARISMENDI, 1989).

De otro lado, según José Luis Romero, Argentina en 1965 atravesaba “tiempos de mucho desconcierto" (ROMERO, 1965b). Palabras que se explican por la frustración dejada por el populismo peronista, seguido de una honda crisis económica, inflación, varios golpes de Estado, gobiernos dictatoriales y un breve retozo democrático, seguido de otro golpe de Estado (ROMERO, 1997). De nuevo, en las elecciones de 1963, llegó a la presidencia Arturo Illia, quien gobernó en medio de una crisis política y se preveía una nueva arremetida de los jefes militares para recuperar el gobierno, en alianza con empresarios y sindicatos. $Y$ en efecto, un año después de la visita de Borges a Colombia, los comandantes de las Fuerzas Armadas depusieron a Illia e instauraron en la presidencia al general Juan Carlos Onganía (ROMERO, 1997).

Como puede verse, el contexto en el que se llevó a cabo el viaje de Borges a 
Colombia se caracterizaba por los momentos difíciles que vivían las democracias de ambos países, en el caso argentino por el auge de gobiernos dictatoriales y breves intentos por restablecer elecciones democráticas, mientras que del lado colombiano hablamos de una democracia restringida, con una hegemonía sostenida de las clases dominantes, amenazada por el crecimiento de las guerrillas que buscaban tomarse el poder. Lo cierto es que, en ambos países, gobernaban presidentes elegidos en las urnas y se intentaba respetar a las constituciones vigentes. En el contexto de la Guerra Fría, las dos naciones orbitaban en torno a los Estados Unidos, y desde el gobierno de John F. Kennedy estrecharon sus relaciones con la potencia norteamericana, contribuyendo en 1962 a excluir a Cuba del sistema interamericano (CAVELIER, 1997).

Las relaciones diplomáticas entre Colombia y Argentina empezaban a despertar en las décadas de 1950 y 1960, circunstancia manifiesta con el encuentro entre los cancilleres de los dos países en Buenos Aires y Bogotá (1960), la participación en la constitución de la Asociación Latinoamericana de Libre Comercio (ALALC) (ROMANO, 2008), y el Tratado Interamericano de Asistencia Recíproca (TIAR) (CAVELIER, 1997). Asimismo, la firma en Bogotá de una Declaración Conjunta de las dos cancillerías y un Convenio de Intercambio Cultural en 1964. Para sellar mejor esta alianza, en 1965 los gobiernos de Arturo Illia y Guillermo León Valencia se declararon anticomunistas y apoyaron la invasión norteamericana a la República Dominicana (TORRES, 1994).

Es oportuna una mirada al comercio binacional y los procesos de migración e inmigración entre ambas naciones. Al respecto, valga decir que en el período de estudio eran escasos los intercambios comerciales, pero el gobierno argentino evidenció interés por proveer de manufacturas a Colombia y comprar materias primas a ésta (HINESTROSA, 1942). También es del caso decir que la balanza comercial estaba a favor de Argentina, y que este país mantuvo un patrón de exportaciones con preferencia a Europa, Estados Unidos y sus países vecinos: Brasil, Uruguay y Chile; mientras que Colombia exportaba la mayor parte de sus productos a Norteamérica y el Viejo continente.

Con respecto a la presencia de ciudadanos argentinos en Colombia, se sabe de individuos y familias dispersas por las principales ciudades, los cuales se trasladaron al país por motivos profesionales, en particular por relaciones culturales e intelectuales 
con sus homólogos colombianos. Ejemplos de la impronta de los argentinos en el arte, la cultura y las letras son numerosos: 1) en el teatro, el dramaturgo Francisco Petrone y los jóvenes actores Fanny Mikey (que luego se nacionalizó colombiana) y Pedro I. Martínez (REYES, 1994), entre muchos otros, como Nélida Quiroga, Luis Linares, Rocco Petruzi, Felipe Sanguinetti, Irma Roy, Rosita Alonso, Elsa Aldao y Eduardo Cuitiño, quienes alternaron sus labores teatrales con actuaciones en la naciente televisión colombiana (REYES, 1994). 2) La crítica de arte Marta Traba, llegó a Colombia en 1954 como profesora universitaria y conferencista, fue fundadora de la Revista Prisma, colaboradora de las revistas Mito y Semana, y en 1962, Directora del Museo de Arte Moderno de Bogotá (MAGLIA, 1994a). 3) Durante el "Dorado" del fútbol colombiano, los equipos del país cafetero recibieron a la élite del balompié argentino: Adolfo Pedernera, Néstor Raúl Rossi, Alfredo Di Stefano, Mario Garelli, Carlos Gambina, Felipe Marino, José Vicente Greco, Américo Montanini, Walter Marcolini y René Pontoni, entre otros (PELÁEZ, 1994).

Por su parte, notables intelectuales colombianos vivieron e hicieron parte de su carrera como escritores, periodistas y diplomáticos en Buenos Aires. Uno de ellos fue Baldomero Sanín Cano, quien estuvo en Buenos Aires en dos períodos (1925-1926 y 1933-1935), participó de cafés, tertulias, eventos y revistas culturales, llegando a ser amigo personal de muchos intelectuales argentinos y colaborador de sus revistas culturales (RUBIANO, 2013; RUBIANO, 2014), así como enviado extraordinario y Ministro Plenipotenciario en Argentina (MEJÍA, 1951). También el historiador Germán Arciniegas estuvo entre 1939 y 1942 cumpliendo funciones de consejero en la embajada colombiana y aprovechó para entablar relaciones con intelectuales argentinos como Victoria Ocampo (ARCINIEGAS, 1994) (amiga en común con Borges) y aprovechó para colaborar en la Revista Sur (MAGLIA, 1994b). Del mismo tenor, merece ser destacado por sus vínculos con Argentina, Jorge Zalamea Borda, quien se radicó en Buenos Aires entre noviembre de 1951 y agosto de 1952, para resguardarse de la persecución en su contra auspiciada por los gobiernos de Mariano Ospina Pérez y Laureano Gómez (ECHAVARRÍA, 1951; ZALAMEA, 1956). Un dato que no debe soslayarse es que Zalamea fue hasta 1960 gran amigo de Arciniegas, y era suegro de Marta Traba.

Evidenciando mayor empeño en las relaciones culturales, académicas y sociales entre Colombia y Argentina, el país austral tuvo la iniciativa, lo que se expresa en la 
diplomacia cultural de la cual hizo parte el viaje de Borges y una delegación argentina, integrada por diplomáticos, escritores e intelectuales, y militares. La presencia de tres notables pensadores colombianos en Buenos Aires -Sanín Cano, Arciniegas y Zalamea- en décadas anteriores, contribuyó por su parte a una mayor visibilidad de la cultura colombiana en el país del sur. Sumado a esto, la fama ganada por Bogotá como la "Atenas suramericana" y el manido "buen uso" del castellano (MAGLIA, 1994b), abonaron el terreno para que en la década de los sesenta fueran más frecuentes los intercambios culturales y académicos entre ambos países. A ello también contribuyeron las relaciones diplomáticas, en particular el convenio de intercambio cultural firmado en 1964, y que los gobiernos de ambas naciones se reconocieran entre sí como democráticos.

\section{De Bogotá a Cali}

El primer viaje realizado de Borges a Colombia fue en 1963 como invitado por el poeta Jorge Gaitán Durán, director de la revista Mito (RINCÓN, 2016), y por la Universidad de los Andes, que le concedió un doctorado honoris causa. El de 1965, en cambio, se debió al gobierno argentino, quien lo integró a una Misión de intercambio cultural, diplomático y militar. La comitiva que acompañó a Borges a Colombia estuvo integrada por su amiga, también conferencista, Esther Zemborain de Torres Duggan, profesora de Filosofía y Pedagogía de la Universidad de Buenos Aires (RAMÍREZ, 1965). $\mathrm{Y}$, además del cuerpo de militares mencionado antes, a cargo de los desfiles para conmemorar las fiestas patrias de ambos países, la delegación argentina estuvo integrada por la declamadora Mara Kelton (SIN AUTOR, 1965d), por el ballet folclórico de Ismael Gómez; y por el quinteto Huancahua, que interpretaba música folclórica del país austral (SIN AUTOR, 1965d).

Los militares visitantes acompañarían los desfiles de Bogotá y Cali, luciendo uniformes de la época de la Independencia (RUEDA, 1965; SIN AUTOR, 1965f) en los desfiles y actos conmemorativos del 20 de julio y el 7 de agosto (SIN AUTOR, 1965f). Otros actos contemplaban la entrega de réplicas del sable corvo del general José de San Martin al presidente de Colombia y a otras autoridades (RUEDA, 1965). Las exhibiciones marciales no se desarrollaron sólo en la capital de la República, sino 
además en el estadio olímpico "Pascual Guerrero" de Cali (SIN AUTOR, 1965f), la participación de sus miembros o comisiones en distintos actos conmemorativos de otras ciudades (RUEDA, 1965). Los militares invitados permanecieron en Cali entre el 10 y el 12 de julio (RAMÍREZ, 1965).

Luego del recorrido de Borges por Bogotá y Cali, se desplazó a Medellín el domingo 11 y el lunes 12 de julio (SIN AUTOR, 1965d; SIN AUTOR, 1965b), de donde partió para Cartagena el 13 julio (SIN AUTOR, 1965d). A la capital del país el argentino llegó en las primeras horas de la noche del miércoles 7 de julio, y fue conducido de inmediato al Teatro Colón donde pronunció una conferencia en torno a "Literatura Argentina de Hoy". Al día siguiente, junto a Esther Zemborain visitó la Universidad de los Andes (ROMERO, 1965a) y en la noche estuvo en el Instituto de Cultura Hispánica exponiendo sobre "La poesía gauchesca" (SIN AUTOR, 1965c), y en el coctel organizado por el embajador argentino y su esposa en la Embajada con asistencia de destacados intelectuales colombianos (POMBO, 1965).

Borges llegó a la capital del Departamento del Valle del Cauca al filo del mediodía del viernes 9 de julio (RAMíREZ, 1965) y allí dictó una conferencia en el Teatro Municipal sobre la "Filosofía del Arrabal" (SIN AUTOR, 1965f). Un día después, en la casa cultural La Tertulia trató el tema de la "Poesía argentina y gauchesca" (SIN AUTOR, 1965f) y ahondó en personajes históricos y populares del país austral (PARDO, 1965). Aunque la gira tenía previsto que el escritor se ausentaría de Cali en la tarde del sábado 10 de julio para dirigirse a Bogotá (RAMíREZ, 1965), ese día en horas de la noche todavía se encontraba en la capital vallecaucana (SIN AUTOR, 1965f). Todo indica que el domingo 11 cuando se desplazaba hacia Medellín acompañado por la Sra. Zemborain, su transporte aéreo realizó una breve escala en la capital de la República (SIN AUTOR, 1965f).

\section{Paso fugaz por Medellín}

En Cali, Borges fue recibido con toda la pompa, por las gestiones del embajador Mathov ante la gobernación del Valle del Cauca con antelación de cinco meses (SIN AUTOR, 1965f), y por ello contó con la presencia del mandatario departamental y de la plana central de su despacho, las autoridades de la ciudad, los mandos militares, el 
rector de la Universidad Santiago de Cali y el cuerpo directivo del Centro ColomboArgentino de Cali. Por contraste, en Medellín Borges fue recibido con respeto, pero sin cúmulo de honores y garbo paralelos. Apenas dos días antes de su llegada la prensa de Medellín informó sobre el arribo del ilustre visitante:

Jorge Luis Borges será desde mañana huésped de Medellín.

Ofrecerá una recepción a los intelectuales y dictará conferencia en el Paraninfo de la Universidad de Antioquia.

El intelectual argentino de renombre internacional y candidato al Premio Nobel, Jorge Luis Borges, llegará mañana a esta ciudad. Será recibido en el aeropuerto Olaya Herrera por sus numerosos admiradores. Viene realizando una gira cultural por el continente americano con el deseo de estrechar los vínculos afectivos entre los escritores de los distintos países.

El domingo, Borges ofrecerá una recepción para intelectuales a quienes hablará sobre cuestiones literarias y sobre los nuevos movimientos del arte en el mundo.

EN LA UNIVERSIDAD

El lunes próximo en el Paraninfo de la Universidad, y dentro del ciclo del "Lunes de las Artes", dictará una conferencia sobre el tema "Literatura de Arrabal".

Esta conferencia está auspiciada, como también su gira por Colombia, por la embajada del país en la Argentina. INVITACIÓN

A la conferencia del lunes en el Paraninfo invitan la Universidad de Antioquia, la asesoría artística, la extensión cultural municipal y el Instituto de Artes Plásticas del Alma Mater (SIN AUTOR, 1965, pp. 4 y 18).

Valga considerar el yerro del titular anterior, al anunciar para el sábado 10 de julio la llegada del personaje, cuando en realidad se produjo, como se ha visto, el domingo 11. En la prensa se le reconoce como "prominente poeta" (BORENSTEIN, 1965a), como eminente cuentista y ensayista (BORENSTEIN, 1965b), destaca su "renombre internacional" y su "candidatura al Premio Nobel de Literatura" (BORENSTEIN, 1965a). Según el anuncio, Borges contaba en Medellín con "numerosos admiradores" dispuestos a recibirlo “en el aeropuerto Olaya Herrera” y afirma que el escritor estaba interesado en estrechar vínculos afectivos con los escritores antioqueños, a quienes hablaría de los "nuevos movimientos del arte en el mundo" (SIN AUTOR, 1965e).

Revisada la información de prensa disponible y considerando la marcada ausencia de profundidad en el despliegue de las noticias, es decir, lo fragmentario de cuanto comunican, todo indica que sus admiradores en Medellín no eran en realidad 
tan "numerosos" como lo manifestó la prensa, ni tampoco tan extenso el círculo intelectual con el que departió sobre "la literatura argentina del momento" (BORENSTEIN, 1965a), en "mesa redonda" efectuada en el Hotel Nutibara junto con “escritores, periodistas, artistas, [y] estudiantes" colombianos, -cuyos nombres no se expresaron públicamente- pero a quienes, se dice, el intelectual viajante les “expuso brillantes ideas", en la noche de aquel domingo tras llegar a la ciudad (SIN AUTOR, 1965a). Merece subrayarse que la noticia que quiso realzar ese notorio despliegue se equivoca de manera protuberante, -cometiendo el mismo desacierto en el que poco antes había incurrido la prensa bogotana (SIN AUTOR, 1965c)-, al indicar que el invitado se llamaba “José” Luis Borges en vez de “Jorge” Luis Borges (SIN AUTOR, 1965a).

Si bien el periódico El Diario destacó que la llegada del escritor a Colombia se debió "a la colaboración que prestó la colonia argentina, residente en Medellín" (BORENSTEIN, 1965a), una revisión más amplia y cotejo sobre lo acontecido en otras ciudades del país, permite asegurar que dicha afirmación se fundamenta en un presupuesto más aventurado que certero, pues no se aviene por completo con la solemnidad de los eventos y la cercanía oficial verificables -por ejemplo- en la ciudad de Cali. Valga recordar cómo el periodista Pardo Llada subrayó que Borges apenas alcanzó a estar en Medellín "un día”, tal vez interpretando con esas dos palabras que pasó de manera fugaz, sobre todo si se atiende al carácter por esencia festivo del domingo, -de suyo ajeno a actividades laborales y culturalmente señalado para el descanso y el cumplimiento de deberes religiosos- en una sociedad marcadamente tradicionalista como la antioqueña.

Se puede precisar, de todos modos, que algunos residentes argentinos -integrantes de una pequeña colonia establecida en ese entonces (HOYOS, 2011)-, caso del Sr. Leonardo Nieto Jarbón, quien había fundado desde 1961 la heladería y pastelería "Versalles", invitaron a su distinguido compatriota a asistir a la ciudad. En concreto el Sr. Nieto convidó a Borges, además, a asistir a su negocio, cortesía gustosamente atendida por el visitante, quien permaneció un agradable rato en el establecimiento (aunque no como lo comenta en su página Web el renombrado restaurante, al afirmar erradamente que el episodio se produjo en 1966, cuando en realidad aconteció en julio de 1965) (SALÓN VERSALLES, 2014). El Sr. Nieto fue de 
hecho uno de los promotores de la visita del escritor a Medellín, tras haber sido contactado para colaborar con ese cometido por el embajador argentino en Colombia. Años después, Nieto tomó parte en varias iniciativas cívicas en la ciudad, como la fundación de la Casa Gardeliana (1973) y del Festival Internacional del Tango (2007) (SIN AUTOR, 2016). Con motivo de la visita de Borges, igual llamado recibió el Sr. Leopoldo Barrionuevo por parte del embajador argentino, y aquél aportó a la iniciativa. Años más tarde continuaría impulsando otros proyectos para difundir la cultura argentina, como lo demuestra su donación a la ciudad de la estatua de Gardel actualmente emplazada en un parque del barrio Manrique (CERVERA, 2010).

El lunes 12 de julio Borges asistió a un almuerzo realizado en su honor en el Club Campestre, donde fue agasajado -de esa manera precisa- por el alcalde municipal Evelio Ramírez Martínez, y los distintos secretarios de su gabinete (FONEGRA, 1965). A diferencia de Cali no parecen haberse producido discursos, cantos de himnos nacionales, presencia de mandos militares, ni grandilocuentes declaraciones. En procura de información fidedigna, y revisados adicionalmente los catálogos del Archivo Histórico de Medellín (AHM) y del Archivo Histórico de Antioquia (AHA), pudo constatarse que no reposan allí registros conocidos y/o disponibles al respecto.

A las 6 p.m. de aquel primer día de la semana (BORENSTEIN, 1965a), en el marco del ciclo "Lunes de las Artes” promovido por la Universidad de Antioquia en su Paraninfo, y atendiendo a una invitación concebida para un público más amplio -que aquella previamente extendida al círculo intelectual de Medellín, ahora formulada in extenso por Extensión Cultural Municipal y el Instituto de Artes Plásticas del Alma Mater (SIN AUTOR, 1965e)-, el destacado visitante pronunció una conferencia dedicada a la "Literatura de Arrabal” (SIN AUTOR, 1965a). Consultado el Archivo Histórico de la Universidad (AHUdeA) no figura información relacionada, aunque a juicio de sus administradores en la época debieron conservarse cintas magnetofónicas como memoria del evento.

La ajetreada historia de la institución educativa que durante las décadas de 1960 y 1970 se vio sacudida por intensas manifestaciones estudiantiles, -igual que otras universidades del país-, hace pensar que dicha información pudo perderse en medio de circunstancias tales como el incendio sufrido por el bloque 16 de Ciudad 
Universitaria el 8 de junio de 1973. Ese día quedaron reducidos a cenizas importantes archivos, cuando después de una asamblea estudiantil -en recordación de nueve compañeros de la Universidad Nacional en Bogotá, caídos el 8 y 9 de junio de 1954 por acción de soldados del dictador Rojas Pinilla (DONADíO, 2007)-, terminó con la muerte a manos de un agente del DAS en inmediaciones del campus, del estudiante Fernando Barrientos. Situación que aquel 8 de junio de 1973 desencadenó disturbios que culminaron con el incendio del bloque administrativo de la Universidad de Antioquia, el toque de queda en la ciudad, el cierre del establecimiento educativo durante dos semanas, y como se ha dicho, la pérdida irrecuperable de documentos (URIBE, 1998).

Cabe recordar que durante los años cercanos a la visita de Borges -y sucesivos-, Colombia experimentó como lo corrobora el historiador Álvaro Tirado Mejía "una época dinámica y de cambios” (TIRADO, 1989), consistente en variaciones económicas y sociales estructurales en un lapso de tiempo relativamente corto: fruto de la urbanización y la industrialización aceleradas, de la aplicación de políticas de control de la natalidad ante el enorme incremento demográfico, de la concentración y tecnificación de la propiedad agraria, del crecimiento de exportaciones legales alternas al café, de variaciones culturales manifiestas en la vinculación femenina al mundo del trabajo, de la crisis de la Iglesia católica y de su ética tradicional, y del ostensible mejoramiento del sistema educativo. Todo ello signado por remanentes de la Violencia política partidista de mediados del siglo, y por la conflictividad social acarreada por los vientos de la Revolución Cubana (1959), esparcidos además sobre el vasto concierto latinoamericano.

En el caso de la Universidad de Antioquia, 1965 fue un año especialmente convulsionado, por cuanto sus estudiantes habían recibido la visita, en marzo, del sacerdote revolucionario Camilo Torres Restrepo, estudiaban marxismo, se mostraban simpatizantes de la Revolución cubana y se declararon en abierta oposición a la rectoría del conservador Ignacio Vélez Escobar. Además, durante el primer semestre protagonizaron graves disturbios contra la invasión estadounidense a la República Dominicana (TORRES DEL RÍO, 1994). Los activistas estudiantiles hacían parte de un consejo estudiantil agremiado a la Federación Universitaria Nacional (creada dos años atrás) y por sus vínculos con los estudiantes de otras universidades públicas lograron que sus protestas se extendieran a otras ciudades del país. Las protestas se 
radicalizaron y enarbolaron otras banderas, como el rechazo a la presencia de misiones norteamericanas y la exigencia de la renuncia del rector Vélez Escobar. En mayo de aquel año las instalaciones del Instituto de Estudios Generales de la Universidad (ubicadas en el centro de la ciudad) fueron allanadas por la policía y con ello el movimiento se radicalizó todavía más, llegando a convertirse en un paro nacional de universidades, con refriegas que incluyeron la muerte de un estudiante en Bogotá. Hasta que intercedió el presidente Guillermo León Valencia, quien le pidió la renuncia al rector de la Universidad de Antioquia, la cual se llevó a cabo para calmar los ánimos estudiantiles y con ello se logró el levantamiento del paro nacional (URIBE, 1998).

Como puede verse, el ambiente político, cultural e intelectual en la universidad antioqueña que fue anfitriona de la conferencia de Borges, se movía entre los extremismos de la izquierda y el confesionalismo cristiano, motivo por el cual, no había un público sensible a la obra literaria del escritor argentino. Seguramente los estudiantes y profesores de la institución pública no estuvieron muy interesados en prestar atenta escucha a un escritor cuyas posturas políticas no simpatizaban con los movimientos de izquierda, que se declaraba crítico de su compatriota Ernesto Guevara y de la Revolución cubana, además de ser agnóstico en materia religiosa y anarquista profundo. Es apenas lógico pensar que el hecho de que la organización del Premio Nobel de Literatura le haya otorgado el galardón al comunista declarado Jean Paul Sartre, negándoselo a Borges, haya sido otro argumento en contra del argentino. Lo cierto es que, luego de rastrear el órgano institucional de ese momento, la Revista Universidad de Antioquia, no se encuentra noticia alguna sobre la visita de Borges o sus obras.

En la revista correspondiente al segundo semestre de 1965 no se encuentra una sola alusión a la conferencia de Borges, pero incluye en sus páginas dos textos del sacerdote Eugenio Lakatos, el primero sobre "La posesión de la Tierra Prometida” y el segundo intitulado "La Santa Biblia". Además, los libros reseñados por la revista fueron: "El Concilio de Trento en su última etapa", "El estudio de la teología”, “Evolución y Biblia” e “Iniciación al ecumenismo”. Aún en el apartado denominado "Vida universitaria", que se dedicaba a las noticias del acontecer educativo y cultural de la institución se ignora el suceso en cuestión, y se resalta la apertura de una 
exposición de libros británicos en la Facultad de Medicina, además de incluir una reseña sobre la medalla al mérito universitario "Francisco Antonio Zea”. En el primer número de 1966 tampoco se alude en lo más mínimo a la visita del ilustre argentino, sino a otros eventos realizados en las instalaciones universitarias, entre otros, el "Encuentro de religiones" en el Paraninfo de la Universidad (UNIVERSIDAD DE ANTIOQUIA, 1966).

No se pretende negar el interés académico de los textos publicados por la revista universitaria, entre los cuales se incluyó una traducción del griego de la tragedia Edipo Rey, un homenaje al sacerdote jesuita Félix Restrepo, fundador de la Universidad Javeriana, y estudios históricos sobre "Santa Rosa de Lima en el espíritu latinoamericano", o "Costa Rica y la guerra contra los filibusteros", sino que es protuberante el silencio de la principal publicación universitaria en torno a la conferencia dictada por Borges en el Paraninfo. Ello refleja claramente el escaso o nulo interés que despertó la presencia del candidato al Nobel de Literatura de 1964, y permite confirmar que su conferencia en la Universidad no estaba articulada a la agenda cultural de la institución. Hasta se puede pensar que, en rigor, los organizadores de la gira sólo consideraron que en vista del significado histórico y cultural de la Universidad y de su Paraninfo, ese era el espacio más digno para el nivel del conferencista, puesto que venía de dictar conferencias y cursos en importantes universidades del mundo.

Por ello no debe extrañar que la primera vez que se publicaron textos de Borges fue en la Separata de Poesía de 1976, donde se incluyó “Ajedrez", "Al hijo", “Amanecer”, “Carnicería” y “Del cielo y el infierno”, es decir once años después de haber dado su charla en el Paraninfo universitario (UNIVERSIDAD DE ANTIOQUIA, 1976). Más aún, el primer escrito crítico y valorativo de la narrativa y la poesía de nuestro personaje en la misma revista sólo se publicó en 1986, es decir veintiún años después de su primera estadía en Medellín.

A manera de epílogo se debe decir que, consumadas las actividades programadas en Medellín, Borges se desplazó a Cartagena el martes 13 de julio de 1965. Desde el momento en que la gira fue proyectada se contempló oficialmente su paso -y el de Zemborain- por esa ciudad, a manera de cierre formal del intercambio cultural. Allí, "en el Paraninfo de la universidad local”, los organizadores dispusieron 
"una serie de conferencias" por parte de los dos escritores. Tan escueta como aquí se enuncia es la información conexa que figura en la prensa, aparte de especificar que, además de la "Ciudad Heroica", también se vería beneficiada con la gira la ciudad de Barranquilla, dado que el domingo 11 y el lunes 12 de julio se presentarían en esta última “el ballet folclórico de Ismael Gómez y el quinteto Huancahua”, grupos integrantes -ya se ha dicho- de la Misión cultural.

\section{Conclusiones}

El estudio del rol como hombre público de un escritor como Borges, a partir de huellas y vestigios documentales, permite conocer de cerca la relación del creador con su público lector, con los gobiernos e instituciones, y destacar facetas que son desconocidas incluso por sus biógrafos más autorizados. Es indudable que no se trató de un viaje de placer o motivado por búsquedas existenciales, sino de una Misión de intercambio cultural, ideada y gestionada en el marco de un convenio diplomático

entre Colombia y Argentina, y cuyos rasgos fueron: celeridad, actos públicos consecutivos y agotadores, pocos o nulos ratos de soledad y sosiego del escritor para consigo mismo, como era del gusto del argentino. Además, resulta consecuente resaltar que, -a diferencia de los otros viajes de Borges a Colombia-, la iniciativa provino del gobierno austral, en cabeza de su ministro de relaciones exteriores, su embajador en Colombia y las colonias establecidas en cuatro de las ciudades visitadas.

Es claro que el conocimiento de la obra borgiana en nuestro país, en particular en Antioquia, se encontraba limitado a una aristocracia de las letras, y que incluso en instituciones como la Universidad de Antioquia, que participó del itinerario de conferencias, eran escasos sus lectores. Lo cual resulta comprensible por tratarse de un año en el que esta universidad estuvo en el centro de las protestas y manifestaciones estudiantiles que llevaron a la renuncia anticipada del rector Ignacio Vélez Escobar, por petición del presidente de la República. Quizás la “macartización” por parte de los sectores de izquierda, quienes consideraban al escritor argentino como un hombre de derecha, y el hecho de no haber recibido, por motivos políticos, el Premio Nobel de Literatura un año antes, cuando se prefirió a un comunista declarado como Jean Paul Sartre, así como el desconocimiento de su obra creativa y 
de sus concepciones políticas -más próximas al anarquismo-, pueden aportar elementos relevantes para explicar que su obra no tuviera difusión en la revista de esta institución universitaria, o que por lo menos dicha difusión fuera muy posterior y tardía.

\section{Referencias}

\section{Artículos de prensa}

BORENSTEIN M. “El Diario Cultural”, en: El Diario, Medellín, Año 35, n. 10.497, julio 9 de 1965 a, p. 4.

BORENSTEIN M. “El Diario Cultural”, en: El Diario, Medellín, Año 35, n. 10.499, julio 12 de 1965 b, p. 4.

ECHAVARRÍA, R. "Cultura, 'Sección especial de Dominical”", en: Magazine Dominical El Espectador, n. 190, Bogotá, 11 de noviembre de 1951, p. 24.

GIRALDO, L. "Una entrevista diferente: La influencia de la política en la literatura es anacrónica", en: Lecturas Dominicales de El Tiempo, Bogotá, agosto 1 de 1965, p. 4.

POMBO, A. “El Día Social. Reuniones para hoy”, en: El Siglo, Bogotá, julio 8 de 1965, p. 6.

RAMÍREZ. "Delegación Argentina Hará Visita a Cali. La encabeza el escritor Jorge Luis Borges”, en: El Tiempo, Bogotá, Año 55, n. 18.683, julio 6 de 1965, p. 8.

ROMERO DE NOHRA, F. "Un Gran Escritor Latinoamericano: Borges Reivindica la Poesía Popular Argentina”, en: El Tiempo, Bogotá, Bogotá, año 55, n. 18.684, julio 7 de 1965a, p. 5.

RUEDA, “Granaderos Argentinos Llegan hoy”, en: El Tiempo, Bogotá, Bogotá, año 55, n. 18.684, julio 7 de 1965, pp. 1,11.

SIN AUTOR, "Conferencia de Borges en el Paraninfo", en: El Diario, Medellín, Año 35, n. 10.499, julio 12 de 1965a, p. 1.

SIN AUTOR, “El Día Cultural. Borges en Medellín”, en: El Siglo, Bogotá, julio 11 de 1965 b, p. 8.

SIN AUTOR, “El Día Cultural. Mes Colombo-Argentino”, en: El Siglo, Bogotá, julio 8 de 1965 c, p. 8.

SIN AUTOR, “Jorge Luis Borges Irá Hoy a Cartagena”, en: El Tiempo, Bogotá, Año 55, n. 18.690, julio 13 de $1965 d$, p. 25.

SIN AUTOR, “Jorge Luis Borges Será Desde Mañana Huésped de Medellín”, en: El Colombiano, Medellín, julio 9 de 1965e, pp. 4 y 18.

SIN AUTOR, "Sección Editorial. Conferencias en el Teatro Municipal y en la Tertulia Dicta Hoy Jorge L. Borges”, en: El Siglo, Bogotá, julio 11 de 1965r, p. 4.

\section{Revistas}

PARDO, J. Preguntó Jorge Luis Borges en Cali: ¿Todavía hay alguien que lea la 'María’?. Cromos, p. 10, ago. 1965

ROMANO, S. Brasil, Argentina y la integración regional durante la década de 1960 en el marco de las relaciones con Estados Unidos. CONfines de Relaciones Internacionales 
y Ciencia Política, v. 4, n. 8, ago. 2008.

RUBIANO, R. Baldomero Sanín Cano en La Nación de Buenos Aires (1909-1957). Prensa, diplomacia y análisis político internacional. Historia y Sociedad, n. 25, 79-105, 2013. RUBIANO, R. Baldomero Sanín Cano y la Revista Nosotros de Buenos Aires (1907-1943). Intercambio y redes culturales e intelectuales. Historia y Sociedad, v. 41, n. 1, 127-156, 2014.

UNIVERSIDAD DE ANTIOQUIA. Separata de Poesía. Revista Universidad de Antioquia, v. 51, n. 196, p. 130-135, ene. 1976.

UNIVERSIDAD DE ANTIOQUIA. Revista Universidad de Antioquia, n. 162, ene.-jun. 1966.

\section{Libros}

ARIZMENDI, I. Presidentes de Colombia 1810-1990. Bogotá: Planeta, 1989.

CAVELIER, G. Política internacional de Colombia. Bogotá: Universidad Externado, 1997.

GUZMÁN, G., FALS, O., y UMAÑA, E. La Violencia en Colombia. Bogotá: Tercer Mundo, 1962 y 1964.

MEJÍA R. Vidas y empresas de Antioquia. Medellín: Imprenta Departamental de Antioquia, 1951.

OSORIO, J., y BUENO, C. Borges. Memoria de un gesto. Medellín: Publicaciones Técnicas, 1979.

OSORIO, J., y BUENO, C. Borges. Memoria de un gesto. Medellín: Instituto Tecnológico Metropolitano, 2003.

ROMERO, J. Breve historia de Argentina. Buenos Aires: Fondo de Cultura Económica, $1965 \mathrm{~b}$.

ROMERO, J. Breve historia de Argentina. Buenos Aires: Tierra Firme, 1997.

Artículos en libros colectivos

ARCINIEGAS, G. "Encuentros culturales", en: Colombianos y argentinos -Historias y encuentros-. Bogotá: Asociación Argentina en Colombia, 1994.

DONADíO, A. “Gobierno de Gustavo Rojas Pinilla (1953-1958)", en: Wills Franco, Fernando (Dir.). Gran Enciclopedia de Colombia, Vol. 3, Bogotá, Casa Editorial El Tiempo-Círculo de Lectores, 2007.

MAGLIA, G. “Arte”, en: Colombianos y argentinos -Historias y encuentros-. Bogotá: Asociación Argentina en Colombia, 1994a.

MAGLIA, G. "Literatura”, en: Colombianos y argentinos -Historias y encuentros-. Bogotá: Asociación Argentina en Colombia, 1994b.

PELÁEZ, H. "Presencia de Argentina en nuestro desarrollo deportivo", en: Colombianos y argentinos -Historias y encuentros-. Bogotá: Asociación Argentina en Colombia, 1994.

REYES, C. "Teatro", en: Colombianos y argentinos -Historias y encuentros-. Bogotá: Asociación Argentina en Colombia, 1994.

TIRADO, Á. "Del Frente Nacional al momento actual: diagnóstico de una crisis", en: Tirado Mejía, Álvaro. (Ed). Nueva Historia de Colombia, Vol. II, Santafé de Bogotá, Planeta, 1989.

TORRES DEL RÍO, C. "Siglo XX: relaciones y políticas", en: Colombianos y argentinos -Historias y encuentros-. Bogotá: Asociación Argentina en Colombia, 1994.

URIBE DE HINCAPIÉ, M. "El movimiento estudiantil: de la lucha por la inclusión a la lucha por el cambio político", en: Uribe de H., María Teresa (Coord. académica). 
Universidad de Antioquia, historia y presencia. Medellín: Editorial Universidad de Antioquia, 1998.

Archivos

Archivo Jorge Zalamea Borda (AJZB)

ZALAMEA, J. Carta de Jorge Zalamea al escritor "Monsieur Vercors" (Seud. de Jean Bruller), Viena, 27 de febrero de 1956.

Archivo Histórico de Medellín (AHM)

FONEGRA, J. "La alcaldía ofrece almuerzo al escritor argentino Jorge Luis Borges", en: Radioperiódico Clarín, Medellín, julio 12 de 1965, sp.

Cibergrafías

CERVERA, A. "Conmemoran 75 años de la muerte de Carlos Gardel en Medellín", en: Archivo El Tiempo, Medellín, 24 de junio 2010, Disponible en: https://www.eltiempo.com/archivo/documento/CMS-7770548. Accedida el 31 de ago.2020.

ECHEVERRÍA, D. “La copa de Borges”, Universocentro, n. 42 (2013). Disponible en: http://www.universocentro.com/NUMERO42/LacopadeBorges.aspx. Accedida el 31 de ago.2020.

ABAD, H. "Borges: 25 años de inmortalidad", en: Héctor Abad Faciolince. Periodismo, 14 de junio de 2016, http://www.hectorabad.com/borges-25-anos-de-inmortalidad/ Accedida el 31 de ago.2020.

RESTREPO, E. “Borges en Medellín: dos textos", en: NTC...documentos, 27 de junio de 2011, Disponible en: http://ntc-documentos.blogspot.com.ar/2011 0627 archive.html. Accedida el 31 de ago.2020.

RINCÓN, J. “El sueño que Borges dejó en Colombia”, en: El Espectador, Bogotá, 13 de junio de 2016. Disponible en: https://www.elespectador.com/noticias/cultura/el-sueno-borges-dejo-colombia-artic ulo-637511. Accedida el 31 de ago.2020.

SALÓN VERSALLES. "Historia. Nuestra cronología”, en: versallesmedellin.com, Medellín, 2014. Disponible en: https://versallesmedellin.com/historia/. Accedida el 31 de ago.2020.

SIN AUTOR. “Homenaje a Don Leonardo", en: Centrópolis. El periódico del centro de Medellín, Medellín, 10 de agosto de 2016, Disponible en: https://www.centropolismedellin.com/homenaje-a-don-leonardo/. Accedida el 31 de ago.2020.

Recebido: 02 set 2020

Aceito: 30 mar 2021 